\title{
Resistencia de bacterias respiratorias y entéricas a antibióticos
}

Fortino Solórzano-Santos, M.C., ${ }^{(1)}$ Ma. Guadalupe Miranda-N ovales, M.C. ${ }^{(2)}$

$L$ as enfermedades respiratorias y diarreicas ocupan los primeros lugares de morbilidad y mortalidad en los países en vías de desarrollo. Debido a la frecuencia con que se presentan estas enfermedades, la prescripción y la automedicación de antibióticos para las mismas se ve favorecida. Se ha considerado que el uso inapropiado o excesivo de antibióticos genera la aparición de cepas resistentes, fenómeno que se ha hecho muy evidente en el ámbito hospitalario, ${ }^{1}$ a pesar de que existen guías o normas para el manejo de los mismos. ${ }^{2}$ Aunado a ello, la resistencia bacteriana secundaria al uso indiscriminado de antibióticos para el tratamiento de infecciones comunes ha sido poco investigada.

Levin y colaboradores ${ }^{3}$ concluyeron en un estudio que con la cesación del uso de los antibióticos se reduciría la frecuencia, la diseminación y la evolución de plásmidos y genes mediadores de resistencia. Sin embargo, en la práctica clínica esto no es viable, ya que los antibióticos no pueden dejar de usarse. Es innegable que al administrar un antibiótico, además de que se actúa contra el patógeno supuesto, también se afecta a los gérmenes comensales de la nasofaringe y el intestino, así como a otros hábitats bacterianos presentes en el humano. A partir de un modelo matemático se ha demostrado la influencia que puede tener un antibiótico sobre la genética de poblaciones bacterianas y su resistencia a antibióticos. ${ }^{3}$ Ello permite sugerir que, a pesar de que se haga un juicioso uso de los antibióticos, la disminución de los porcentajes de resistencia en poblaciones bacterianas comensales y patógenas es moderada; inclusive, si se deja de usar un antibiótico, no es de esperarse que las bacterias regresen a los niveles de sensibilidad del pasado. Por lo anterior, la única medida para retrasar la multirresistencia bacteriana es el uso prudente de los antibióticos.

En este trabajo nos proponemos describir el comportamiento de la resistencia bacteriana a los antibióticos en los principales patógenos que causan infecciones respiratorias y entéricas, destacando algunos aspectos esenciales en cada grupo bacteriano. Por la extensión del manuscrito no se describirán los detalles finos sobre los mecanismos de esta resistencia.

Entre las bacterias de importancia clínica que con mayor frecuencia causan infecciones respiratorias destacan, en los casos de infecciones respiratorias altas, el Streptococcus pyogenes y, en los de infecciones respiratorias altas y bajas, el Streptococcus pneumoniae y el Haemophilus influenzae.

\section{Streptococcus pyogenes (Streptococcus beta hemolítico del grupo A)}

Tradicionalmente se ha considerado que estos estreptococos mantienen una elevada sensibilidad a los viejos y nuevos antibióticos; sin embargo, existen algunas evidencias de fallas bacteriológicas. A los clínicos les ha inquietado la posibilidad de que se generen cepas de estreptococo beta hemolítico del grupo A (SGA) resistentes a los antibióticos, particularmente por el resur-

(1) Departamento de Infectología, Hospital de Pediatría, Centro Médico N acional siglo XXI (CMN Siglo XXI), Instituto Mexicano del Seguro Social (IMSS), México.

(2) Unidad de Investigación en Epidemiología Hospitalaria, Hospital de Pediatría, CMN Siglo XXI, IMSS, México.

Solicitud de sobretiros: Dr. Fortino Solórzano Santos. Departamento de Infectología, Hospital de Pediatría, Centro Médico N acional Siglo XXI, Instituto Mexicano del Seguro Social. Av. Cuauhtémoc 330, Col. Doctores, 06720 México, D.F., México. 
gimiento de infecciones graves y de complicaciones supurativas (síndrome de choque tóxico, fascitis necrosante) y no supurativas (fiebre reumática).

Desde la creación de la penicilina, los SGA presentaron una excelente susceptibilidad ante la misma, de tal modo que se notificaron porcentajes de curación superiores a $90 \%$; las fallas para erradicar los microrganismos de las vías respiratorias altas eran muy raras hasta la década de los setenta, cuando se describieron casos de falla bacteriológica durante los tratamientos. ${ }^{4}$ Por esta época se llegaron a describir cepas mutantes producidas en el laboratorio, pero que no tenían ningún significado clínico. ${ }^{5}$ Los últimos estudios, que han incluido una serie grande de aislamientos, demuestran que los SGA son altamente susceptibles a la penicilina $\mathrm{G}$ con una $\mathrm{CMI}_{90}$ de $0.012 \mu \mathrm{g} / \mathrm{ml}$ y que menos de $5 \%$ de las cepas son resistentes a la eritromicina. Otros macrólidos (azitromicina y claritromicina) mostraron también muy buena actividad bacteriana in vitro pero, por otra parte, se requirieron mayores concentraciones de quinolonas (ciprofloxacina). ${ }^{6}$ Los sistemas de vigilancia sobre resistencia bacteriana en el ámbito mundial han demostrado que en la actualidad aún no existen cepas de SGA resistentes a penicilina, la cual continúa siendo el fármaco de elección. En las infecciones sistémicas graves, donde se ha demostrado o se sospecha la participación de SGA, se sugiere la asociación con otros antimicrobianos. El uso inapropiado de cefalosporinas orales de segunda o tercera generación favorece la inducción de resistencia en la flora comensal, y el beneficio real que éstas aportan en el manejo de infecciones respiratorias altas no es superior al de la penicilina natural.

\section{Streptococcus pneumoniae}

Las infecciones causadas por S. pneumoniae son un problema de salud tanto en países desarrollados como en aquellos en vías de desarrollo. En Estados Unidos de América (EUA) estos patógenos son causa de alrededor de 500000 casos por año de neumonías comunitarias con una letalidad de 5\%. ${ }^{7}$ En México, las neumonías son la segunda causa más común de mortalidad entre niños menores de cinco años (290.5 casos / 1000 personas en menores de un año y 17.2/1 000 en niños de 1 a 4 años); $;$ alrededor de $50 \%$ de los casos presentan neumonías de origen bacteriano, y los agentes más comunes son S. pneumoniae y H. influenzae.

Desde que se empezó a emplear la penicilina, las cepas de $S$. pneumoniae se mostraron muy sensibles a ella, pues para erradicarlas sólo se requerían concentraciones inhibitorias menores a $0.01 \mu \mathrm{g} / \mathrm{ml}$, por lo que este antibiótico fue considerado como el fárma- co de elección. El primer aislamiento clínico de S. pneumoniae resistente a penicilina se notificó en 1967, en Papua, Nueva Guinea, y durante los siguientes 10 años hubo informes esporádicos de casos clínicos con cepas resistentes a peniclina en diversas partes del mundo. A partir de 1977 se detectó un brote de infecciones por neumococos multirresistentes en Sudáfrica. ${ }^{9}$ Más adelante, Soares informó de la diseminación de una clona multirresistente desde España en la década de los años ochenta, ${ }^{10}$ y Muñoz y colaboradores señalaron la evidencia de una diseminación intercontinental de una clona del serotipo 23F ${ }^{11}$ multirresistente y con alta resistencia a la penicilina.

En México, desde 1981, fueron notificadas las primeras cepas resistentes in vitro, con una concentración mínima inhibitoria (CMI) de entre 1.25 y $2 \mu \mathrm{g} / \mathrm{ml}^{12}$ aisladas de niños sanos. En 1993 se informó sobre una resistencia de $43.2 \%$ en portadores asintomáticos de una guardería, y de $12.8 \%$ en niños hospitalizados. ${ }^{13}$ Como parte de un sistema nacional de vigilancia regional, se ha podido evaluar un número representativo de cepas, cuyo origen se ha ubicado predominantemente en niños enfermos hospitalizados; en estos casos se encontró que $16 \%$ de los aislamientos a partir de líquido pleural y aspirado bronquial tuvieron alta resistencia (es decir, una CMI mayor a $2 \mu \mathrm{g} / \mathrm{ml}$ de penicilina), y alrededor de $20 \%$, una resistencia intermedia. ${ }^{14}$ En este mismo informe, $13 \%$ de las cepas fueron resistentes a eritromicina; $43 \%$, a cloranfenicol, y $10 \%$, a cefotaxima. Por otra parte, en portadores nasofaríngeos de una población rural del estado de Tlaxcala, México, $3 \%$ de los S. pneumoniae tuvieron alta resistencia, y $12.6 \%$, resistencia intermedia, con bajos porcentajes de resistencia para el cloranfenicol $(4 \%)$, la cefotaxima $(1.5 \%)$ y la ceftriaxona (3\%). El principal mecanismo de resistencia de $S$. pneumoniae a la penicilina es la alteración en las proteínas fijadoras de penicilina (PBPs).

De acuerdo con los hallazgos anteriores, la Alianza para el Uso Prudente de Antibióticos (APUA) sugiere que, en los casos de infecciones respiratorias bajas por neumococos con resistencia intermedia, se continúe utilizando penicilina con la recomendación de incrementar la dosis por kilogramo de peso; para las cepas resistentes, la alternativa es el uso de ceftriaxona. ${ }^{15} \mathrm{Con}$ base en nuestros hallazgos, ${ }_{1}^{13}$ tanto en el área rural como en aislamientos de niños hospitalizados con neumonía, consideramos que aún en este momento la penicilina G sódica a $50000 \mathrm{UI} / \mathrm{kg} /$ dosis continúa siendo el fármaco de elección para tratar la neumonía comunitaria. En los casos con evolución no satisfactoria, las alternativas son ceftriaxona o cefotaxima, o bien, vancomicina. 


\section{Haemophilus influenzae}

H. influenzae es una bacteria gramnegativa que causa meningoencefalitis e infecciones de vías aéreas, entre otras enfermedades graves. $H$. influenzae puede ser recuperado con mucha frecuencia de la nasofaringe, la cual representa su hábitat natural; periódicamente, a lo largo de la vida, se adquiere y se pierde el estado de portador. ${ }^{16}$ Las cepas que colonizan la nasofaringe difícilmente son erradicadas por la acción del tratamiento antimicrobiano. Según diversos estudios, la frecuencia de portadores asintomáticos es de alrededor de $30 \%, 17-19$ y en los aislamientos, los $H$. influenzae no tipificables han sido predominantes; también se ha podido encontrar $H$. influenzae tipo b, con una frecuencia menor a $10 \%$.

En forma tradicional se ha considerado que la ampicilina y el cloranfenicol son los fámacos de elección para el manejo de las infecciones causadas por este microrganismo. $\mathrm{Al}$ inicio de la década de los años ochenta, se observó un incremento paulatino en la resistencia antimicrobiana, ${ }^{20,21}$ y hacia el final de la década se presentaron en el mundo varios brotes por cepas multirresistentes; actualmente en España éste es un fenómeno prevalente..$^{22-24}$

En 1981 se encontró en México que 14\% de las cepas de $H$. influenzae tipo b eran resistentes a la ampicilina, y no se hallaron cepas resistentes al cloranfenicol. ${ }^{25}$ Hasta 1986 se notificaron las primeras dos cepas con multirresistencia, fenómeno que continúa siendo esporádico en la actualidad. ${ }^{26}$

En estudios recientes hechos con portadores faríngeos asintomáticos, los porcentajes de resistencia a ampicilina y cloranfenicol han sido variables dependiendo de la población estudiada, pero en general se encuentra alrededor de $20 \%$ de resistencia a ampicilina y una nula o muy baja resistencia al cloranfenicol; en los estudios realizados en México, la resistencia hacia macrólidos ha sido elevada. . $71,1927^{2}$

En general se recomienda utilizar la ampicilina o la amoxicilina como fármaco de primera elección para el tratamiento de la otitis media, ya que son las que garantizan una mejor penetración en el líquido del oído medio. Por otra parte, es conocido que en un alto número de casos de otitis media aguda hay resolución espontánea sin uso de antibióticos, por lo que no se requiere de utilizar otros medicamentos de amplio espectro; en este caso, como fármaco alternativo se tiene el trimetoprim-sulfametoxazol (PMP/SMZ). En casos de neumonía confirmada o sospechosa por $H$. influenzae, se recomienda el uso de cloranfenicol; algunos investigadores, considerando los posibles efectos colaterales del mismo, sugieren utilizar cefalosporinas de segunda generación.

Los mecanismos básicos de resistencia de $H$. influenzae se originan en la producción de betalactamasas y acetiltranferasa del cloranfenicol.

\section{Resistencia antimicrobiana en organismos causantes de enfermedades diarreicas}

En los países en vías de desarrollo las infecciones entéricas se encuentran aún entre las principales causas de muerte, sobre todo en menores de cinco años. El tratamiento antimicrobiano ha aportado un claro beneficio al manejo de algunas enfermedades diarreicas, entre las que se incluyen: shigelosis, cólera, diarrea del viajero (más frecuentemente causada por Escherichia coli enterotoxigénica -ETEC-) y diarrea por Clostridium difficile. Aunque la fiebre tifoidea no es una enfermedad diarreica, en ocasiones puede presentarse como diarrea aguda, y la vía de entrada del agente es el tubo digestivo, por lo que se integra a las enfermedades entéricas. En las infecciones por Salmonella no typhi, la prescripción de antimicrobianos se ha justificado para evitar una progresión de la infección, así como complicaciones severas en pacientes inmunocomprometidos. $^{28}$

La resistencia antimicrobiana es un problema creciente en el tratamiento de las infecciones entéricas. En los países en desarrollo la aparición de microrganismos multirresistentes se facilita debido al mayor número de casos de enfermedades entéricas y al uso de antimicrobianos que no son prescritos por el médico. ${ }^{29}$

Entre los bacilos gramnegativos que causan diarrea, los mecanismos de resistencia bacteriana más comunes son: inhibición enzimática; alteraciones de la membrana de la bacteria, y alteración de sitios blanco como los ribosomas, los precursores de la pared y las enzimas. Existen varios cambios genéticos que pueden presentarse en la evolución microbiana: una mutación puntual que ocurre en un par de bases; un segundo cambio que resultaría en un rearreglo del material genético en grandes segmentos de $\mathrm{ADN}$ en un solo evento, y finalmente, un tercer cambio que involucraría la adquisición de $\mathrm{ADN}$ extraño transportado por plásmidos, bacteriófagos o transposones ${ }^{30}$ Estos cambios permiten a la bacteria adquirir un número ilimitado de mecanismos de resistencia. Una vez que aparece, un gen de resistencia puede diseminarse a otras bacterias por transformación, transducción, conjugación o transposición. Algunas clonas seleccionadas pueden proliferar en la flora de pacientes que reciben antimicrobianos..$^{30-31}$ 


\section{Shigella}

Como consecuencia de la dosis infectante reducida (10 a 100 bacterias de Shigella dysenteriae tipo 1 o unos pocos miles de $S$. flexneri) y su resistencia al $\mathrm{pH}$ ácido del estómago, estas bacterias se transmiten fácilmente por contacto directo de persona a persona. ${ }^{32}$

Shigella puede llegar también a la comida o al agua y diseminarse de manera eficaz en nuevos hospederos. Las especies de Shigella son organismos invasivos que, en las últimas décadas, han mostrado un desarrollo progresivo de resistencia a los antimicrobianos de bajo costo y uso amplio. Alrededor del mundo se han utilizado para su tratamiento sulfonamidas, tetraciclinas, ampicilina, TMP/SMZ y ácido nalidíxico. Los perfiles de resistencia que se han encontrado en Bangladesh son representativos de muchas áreas de países en desarrollo. ${ }^{32}$ La tasa de resistencia ha incrementado con el tiempo, y $S$. dysenteriae tipo I es la especie más problemática, pues durante los años noventa ha presentado porcentajes de resistencia mayores a $60 \%$ para ampicilina, TMP/SMZ y ácido nalidíxico. S. flexneri continúa siendo sensible al ácido nalidíxico. ${ }^{33}$

En 1976 se notificó en México la existencia de brotes epidémicos por $S$. dysenteriae tipo 1 multirresistente, con patrones de resistencia similares a los de brotes ocurridos en otros países de Latinoamérica. ${ }^{34}$ Posteriormente se encontraron, durante los años ochenta, resistencias de 25 a $30 \%$ para ampicilina, cloranfenicol y TMP/SMZ. La sensibilidad fue adecuada para furazolidona y cefalosporinas de tercera generación. ${ }^{35}$ Los perfiles de resistencia son semejantes en la actualidad.

\section{Vibrio cholerae}

El cólera es una enfermedad diarreica aguda, causada por la toxina de Vibrio cholerae, que puede tener una evolución grave relacionada con la pérdida excesiva de líquidos y electrolitos. A partir del inicio de la década de los noventa se ha presentado como una enfermedad común en nuestro país. Como en otras enfermedades diarreicas, las fuentes de infección son el agua contaminada de pozos, riachuelos y cisternas, así como los alimentos contaminados, principalmente las hortalizas y los mariscos. ${ }^{36}$

Aunque lo fundamental en el tratamiento del cólera es la terapia de rehidratación, los antimicrobianos disminuyen la excreción del microrganismo por medio de las heces y la diseminación del mismo. Durante los últimos 30 años, las tetraciclinas han sido el fármaco de elección, aunque también se ha utilizado furazolidona, eritromicina y TMP/SMZ. La resisten- cia que se ha encontrado es mediada por plásmidos que se pierden muy fácilmente; por tanto, los patrones de resistencia no son uniformes. A pesar de que se había encontrado en Bangladesh $V$. cholerae 01 resistente a tetraciclina en porcentajes de 50 y $60 \%$, para 1994, 80\% de los aislamientos eran sensibles. La resistencia para TMP/SMZ es mayor a 70\%; para furazolidona, de 20 a $47 \%$, y para eritromicina, doxiciclina y ciprofloxacina, menor a $5 \% .^{28}$

\section{Escherichia coli enterotoxigénica}

Las cepas de ETEC elaboran dos tipos diferentes de toxinas; la primera es una proteína dimérica de alto peso molecular (86 500), similar en estructura química, función y antigenicidad a la toxina producida por $V$. cholerae 01 ; ésta se inactiva con calor $\left(100{ }^{\circ} \mathrm{C}\right.$ durante $10 \mathrm{~min}$ ) y se denomina termolábil. La otra familia de enterotoxinas producidas por cepas ETEC son las denominadas termoestables (ST) ${ }^{37}$ Esta bacteria es la causa más común de la diarrea del viajero.

Inicialmente, la doxiciclina fue el fármaco de elección, pero con el incremento de cepas de ETEC y Shigella resistentes, TMP/SMZ y, más recientemente, las fluoroquinolonas la han sustituido. Los genes plasmídicos que codifican para resistencia son los mismos que contienen la información de dos enterotoxinas, por lo que el incremento en la resistencia podría estar ligado a la transferencia de los genes de enterotoxinas a otros organismos entéricos. Para casos de diarrea aguda por ETEC en la infancia, no se han demostrado beneficios con el tratamiento antimicrobiano. ${ }^{29}$

En México, en cepas aisladas de pacientes pediátricos durante 1960-1980, la resistencia para la ampicilina se incrementó de 35 a 80\%; para la tetraciclina, se mantuvo en más de $90 \%$; para el cloranfenicol, aumentó de 57 a $68 \%$, y para el TMP/SMZ, de 52 a $72 \% .{ }^{38}$

\section{Clostridium difficile}

Es un microrganismo oportunista que, en condiciones normales, es inhibido por componentes de la flora normal, como los enterococos, los lactobacilos y algunas cepas de E.coli. Cuando este microambiente se altera, C. difficile coloniza y se multiplica principalmente en el colon. Puede producir una diarrea acuosa benigna, colitis inespecífica o colitis pseudomembranosa. La diarrea se presenta generalmente relacionada con el uso de antimicrobianos de amplio espectro. Las áreas alrededor de un paciente con diarrea o colitis son contaminadas de modo habitual por el bacilo, lo mismo que las manos del personal médico y paramédico, lo que puede ocasionar brotes intrahospitalarios. ${ }^{39}$ Los 
fármacos utilizados en el tratamiento son vancomicina y metronidazol; sin embargo, ya se ha notificado resistencia al primero, por lo que se deben buscar nuevas alternativas terapéuticas. ${ }^{40}$

\section{Campylobacter jejuni}

A pesar de que Campylobacter es susceptible a la acción bactericida del ácido gástrico, la ingestión de la bacteria en vehículos que mantienen un rápido tránsito por el estómago -como el agua y la leche- y que, por tanto, evaden la barrera natural de la acidez gástrica, permite el paso de la bacteria al intestino. El periodo de incubación varía de 1 a 7 días, y los pacientes excretan de $10^{6}$ a $10^{9}$ Campylobacter por gramo de materia fecal; si no son tratados, la excreción puede llevarse de 2 a 3 semanas. ${ }^{41}$

La infección entérica por Campylobacter es semejante a la causada por Salmonella. Se trata de una zoonosis y se encuentra en las vías gastrointestinales de una gran variedad de animales. Hay varios mecanismos de transmisión bien definidos, incluyendo la ingestión de agua y alimentos de origen animal contaminados -como aves y otras carnes mal cocidas, y leche sin pasteurizar-, así como el contacto con animales, entre los que se encuentran las mascotas. La enfermedad por este microrganismo no es tan severa, aun cuando éste sea invasivo.

En países desarrollados la incidencia de casos de diarrea por este germen es mayor en niños de 0 a 5 años y en adultos de 21 a 30 años. En países en desarrollo, la prevalencia de diarrea por este microrganismo es mayor, aunque la infección se presenta casi exclusivamente en niños. La forma más común de transmisión es la ingestión de agua o alimentos contaminados con la bacteria, y las epidemias de Campylobacter notificadas en estos países se han asociado al consumo de leche o de agua para beber. ${ }^{41}$

Algunos estudios han demostrado que el tratamiento antimicrobiano puede disminuir el periodo de enfermedad. Se ha demostrado que eritromicina, tetraciclina, furazolidona y clindamicina son útiles en su tratamiento. ${ }^{42}$ Sin embargo, su identificación en el laboratorio no es tan rápida como para permitir el inicio de un tratamiento temprano. Recientemente se han descrito cepas resistentes a fluoroquinolonas, eritromicina y tetraciclinas. ${ }^{42}$

\section{Salmonella typhi}

Existen varios fármacos disponibles para el tratamiento de la fiebre tifoidea. El cloranfenicol continúa siendo utilizado ampliamente, y como alternativas están la ampicilina y el TMP/SMZ. Sin embargo, se ha observado un incremento en la resistencia durante los últimos cinco años, con porcentajes cercanos a $40 \%$ para las tres drogas mencionadas; en estos casos es necesario utilizar fluoroquinolonas para el tratamiento de la enfermedad. ${ }^{43}$

En los años setenta se identificó en México y Centroamérica un brote debido a $S$. typhi multirresistente. La resistencia a cloranfenicol en México, durante 1972, fue mayor a $75 \%$, y más de $92 \%$ de las cepas resultaron resistentes, además, a tetraciclina, estreptomicina y sulfonamidas. Posteriormente hubo un franco descenso en la resistencia para ampicilina, cloranfenicol y TMP/SMZ (5-10, 5 y 10\%, respectivamente), el cual se ha mantenido. ${ }^{35,38}$

\section{Salmonella no typhi}

Entre 1960 y 1987, en un hospital pediátrico de México se encontró que la resistencia de Salmonella incrementó para ampicilina (7vs 30\%) y tetraciclina (26 vs 42\%). La resistencia a TMP/SMZ se mantuvo menor a $15 \%$, y al igual que para Shigella, la furazolidona demostró ser un fármaco altamente efectivo. ${ }^{38}$ Sin embargo, en otro centro pediátrico, durante 1982-1985 la resistencia para ampicilina, cloranfenicol y TMP/SMZ fue muy elevada (30-50\%), lo que se atribuyó a la existencia de brotes epidémicos en el hospital. ${ }^{35,38}$

\section{Salmonella entérica serotipo tiphimurium}

En años recientes una cepa de Salmonella conocida como tipo 104 (DT 104) ha cobrado importancia debido a su multirresistencia. Los primeros casos se notificaron en el Reino Unido, y durante los últimos cinco años este microrganismo se ha encontrado con frecuencia creciente en EUA. Esta cepa es resistente a ampicilina, cloranfenicol, estreptomicina, sulfonamidas y tetraciclina. La decisión de utilizar un tratamiento antimicrobiano en casos de infección por Salmonella no typhi depende de la severidad de la enfermedad. Este patrón de multirresistencia debe tomarse en cuenta al decidir los esquemas terapéuticos en caso de enfermedades invasivas; por lo general, se recomiendan las fluoroquinolonas o una cefalosporina de tercera generación como la ceftriaxona. ${ }^{44}$

Para disminuir este problema de resistencia es necesario el uso prudente de antimicrobianos en la comida de animales y limitar, sobre todo, los fármacos que se empleen en humanos para el tratamiento de enfermedades. El descubrimiento de nuevos medicamentos hace parecer que estamos un paso adelante de las bacterias; sin embargo, en muchos casos, la evolu- 
ción de estas últimas ha sido más rápida, por lo que la efectividad de un fármaco contra ciertos patógenos específicos se ha ido limitando. No hay duda de que los nuevos antimicrobianos han permitido disminuir la morbilidad y la mortalidad humanas; sin embargo, para maximizar estos beneficios, nos corresponde establecer la vigilancia del uso adecuado de los antimicrobianos y cuidar minuciosamente la forma en que son prescritos.

\section{Referencias}

1. Goldmann DA, Huskins W C. C ontrol of nosocomial antimicrobial-resistant bacteria: A strategic priority for hospitals worldwide. Clin Infect Dis 1997;24 Suppl 1:S139-S145.

2. Lomas J,Anderson GM, D omnick-Pierre K,Vayda E, Enkin MW, Hannah W J. D o practice guidelines guide practice?The effect of a consensus statement on the practice of physicians. N Engl J Med 1989;321:1306-1311. 3. Levin BR, Lipsitch M, Perrot V, Schrag S, Antia R, Simonsen L et al.The population of genetics of antibiotic resistance. $C$ lin Infect $D$ is 1997;24 suppl 1:S9-S16.

4. Kaplan EL. Benzathine penicillin $\mathrm{G}$ for treatment of group A streptococal pharyngitis: A reppraisal in 1985. Pediatr Infect D is J 1985;4:592-596. 5. Gutmann L,Tomaz A. Penicillin-resistant and penicillin-mutants of group A streptococci. Antimicrob A gents Chemother 1982;2:128-136.

6. Coonan KM, Kaplan EL. In vitro susceptibility of recent $\mathrm{N}$ orth American group A streptococcal isolates to eleven oral antibiotics. Pediatr Infect $D$ is J 1994;13:630-635.

7. Breiman RF, Spika JS, N avarro VJ, D arden PM, D asby CP. Pneumococcal bacteremia in Charleston County, South Carolina: A decade later. Arch Intern Med 1990;150:1401-1405.

8. Sistema $\mathrm{N}$ acional de Evaluación. Tercera Vigilancia de los Progresos en la Aplicación de Estrategias de Salud para Todos en el año 2000, SPT/ 2000. México, D.F.: IN EG I/D GEIE, 1994.

9. Zighelboim S, Tomaz A. Penicillin-binding proteins of multiply antibiotic-resistant South African strains of Streptococcus pneumoniae. Antimicrob Agents Chemother 1980;17:434-442.

10. Soares S, Kristinsson KG , Musser JM, Tomaz A. Evidence for the introduction of a multiresistant clone of serotype $6 \mathrm{~b}$ Streptococcus pneumoniae from Spain to Iceland in the late 1980s. J Infect D is 1993;168:158-163.

11. Muñoz R, Coffey TJ, Daniels M, Dowson CG, Laible G, Casal J et al. Intercontinental spread of a multiresistant clone of serotype 23F Streptococcus pneumoniae. J Infect Dis 1991;164:302-306.

12. G uiscafré GH, G arcía MM, Trejp PJA, G arcía M, Hernandez VR, Muñoz $H O$. Frecuencia de Haemophilus influenzae y de Streptococcus pneumoniae resistente a penicilina en portadores sanos. Arch Invest Med Mex 1981; 12:141-151.

13. Calderón JE, Echaniz AG, Conde GC, Rivera SR, Barriga AG, Solórzano SF et al. Resistencia y serotipificación de 83 cepas de Streptococcus pneumoniae aisladas de niños portadores asintomáticos y enfermos. Bol Med Hosp Infant Mex 1993;50:854-860.

14. Echaniz $A G$, Velázquez $M M, C$ arnalla BMN, Soto NA, Solórzano SF, $G$ atica MR et al. Antimicrobial susceptibilities and capsular types of invasive Streptococcus pneumoniae isolated in children in Mexico City. Microb Drug Resist 1997;3:153-157.

15. Low DE, Azavedo J, McG eer A. El impacto de los neumococos resistentes a penicilina en la práctica clínica. Enferm Infecc Microbiol Clin 1998:18:137-140.
16. Moxon RE. The carrier state: Haemophilus influenzae. J Antimicrob Chemother 1986;18:17-24.

17. Miranda N MG, Solórzano SF,Velázquez AR, Leaños MB,Villasis KMA, Guiscafré GH. Características de los aislamientos de Haemophilus influenzae de portadores asintomáticos en edad pediátrica y su relación con resistencia a antimicrobianos. Bol Med Hosp Infant Mex 1995;52:148-153. 18. Anianson $G, A$ Im B, Andersson B, Larsson $P$, N ylen 0 , Peterson $H$ et al. $N$ aso pharyngeal colonization during the first year of life. J Infect $D$ is 1992;165 supl 1:38-42.

19. Villaseñor SA, Avila FC, Santos PJI. Impacto de las infecciones por Haemophilus influenzae en niños mexicanos. Bol Med Hosp Infant Mex 1993;50:415-421.

20. Doern G, Jorgensen J,Thornsberry C, Preston DP, Tubert T, Redding JS et al. $N$ ational colaborative study of prevalence of antimicrobial resistance among clinical isolates of Haemophilus influenzae. Antimicrob Agents Chemother 1988;32:180-185.

21. Campos J, García TS, Sanfeliu S. Susceptibility studies of multiply resistant Haemophilus influenzae isolated from pediatric patients and contacts. Antimicrob Agents Chemother 1984;25:706-709.

22. Jorgensen J. Update on mechanism and prevalence of antimicrobial resistance in Haemophilus influenzae. Clin Infect D is 1992;14:1119-1123. 23. Scott GM, Thompson R, Rebec MP, Kibbler CC, Smith MD, Holton J. 0 utbreaks of multirresistant Haemophilus influenzae infection. Lancet 1990;1:925

24. Campos J, Garcia TS, Gairi JM, Fábregues Y. Multiply resistance Haemophilus influenzae type $b$ causing meningitis: Comparative clinical and laboratory study. J Pediatr 1986;108:897-902.

25. Trejo y Perez JA, Guiscafré GH, García M, Jaime CM, González $S$, Muñoz 0 . Sensibilidad de Haemophilus influenzae a ampicilina o cloranfenicol en niños de la ciudad de México. Bol Med Hosp Infant Mex 1981;38: 79-85.

26. G uiscafré $H$, Solórzano SF, D elgado 0 , Muñoz 0 . Haemophilus influenzae type $\mathrm{b}$ meningitis resistant to ampicillin and chloramphenicol. Arch D is Child 1986;61:691-707.

27.W einberg GA, Spitzer ED, Murray PR.Antimicrobial susceptibility patterns of $\mathrm{Haemophilus} \mathrm{isolates} \mathrm{from} \mathrm{children} \mathrm{in} \mathrm{eleven} \mathrm{developing} \mathrm{nations.}$ Bull W orld Health O rgan 1990;68:179-184.

28. Sack RB, Rahman M, Yunus M, Khan EH. Antimicrobial resistance in organisms causing diahrreal disease. Clin Infect $D$ is 1997;24 suppl 1: S102-S105.

29. Holmberg SD, Solomon SL, Blake PA. Health and economic impacts of antimicrobial resistance. Rev Infect D is 1987:9:1065-1078.

30. Lupski JR. Molecular mechanisms for transposition of drug-resistance genes and other movable genetic elements. Rev Infect D is 1987; 9:357-368.

31. Mayer KH, O pal SM, Medeiros AA. Mechanisms of antibiotic resistance. En: Mandell GL, Bennet JE, D olin R, eds. Principles and practice of infectious diseases. 4a. edición. N ueva York: Churchill Livinsgtone, 1995: 212-224.

32. Bennish ML, Wojtyniak BJ. Mortality due to shigellosis: Community and hospital data. Rev Infect D is 1991;13:S245-S249.

33. Bennish ML, Salam MA, H ossain MA, Myaux J, Khau EH, Chakrabaty J et al. Antimicrobial resistance of Shigella isolates in Bangladesh, 19831990: Increasing frequency of strains multiply resistant to ampicillin, trimethoprim-sulfamethoxazole and nalidixic acid. Clin Infect D is 1992;14: 1055-1060.

34. G onzález-C ortés A, Heredia-D uarte A, G uzmán J, Ruiz G, Hernández Arreourtua $H$. Epidemia de tifoidea por cepas cloranfenicol resistentes en México en 1972. Estudios de transmisión y vigilancia. Salud Publica Mex 1974:34:37-39.

35. Solórzano SF, Leaños MB, Guiscafré GH. Resistencia antimicrobiana actual de Salmonella typhi, Salmonella enteritidis y Shigella sp. Bol Med Hosp Infant Mex 1987:44:448-455. 
36. Kumate J, Sepúlveda J, Gutiérrez G. El cólera. Epidemias, endemias y pandemias. México, D.F.: N ueva Editorial Interamericana, 1993.

37. Levine MM. Escherichia coli that cause diarrheae: Enterotoxigenic, enteropathogenic, enteroinvasive, enterohemorrhagic and enteroadherent. J Infec D is 1987;155:377-389.

38. Santos JI, De la Maza L, Tanaka J. Antimicrobial susceptibility of selected bacterial enteropathogens in Latin America and W orldwide. Scand J Gastroenterol 1989;24 suppl 169:28-33.

39. Camorlinga-Ponce M, Gamboa M, Barragan JJ, Muñoz O, Fekety FR, Torres JF. Epidemiological aspects of Clostridium difficile in a pediatric hospital and its role in diarrhoeal disease. Eur J Clin Microbiol Infect Dis 1987;6:542-544.

40. D worczynski A, Sokol B, Meisel-Mikolajczyk F.Antibiotic resistance of Clostridium difficile isolates. Cytobios 1991;65:149-153.
41. Riley IW, Finch MJ. Results of the first year of national surveillance of Campylobacter infections in the United States. J Infect D is 1985;147: 243-245.

42. Salazar-Lindo E, Sack RB, Chea-W oo E, Kay BA, Piscoya ZA, LeónBarva $P$ et al. Early treatment with erythromycin of Campylobacter jejuni associated dysentery in children.J Pediatr 1986;109:355-360.

43. Threlfall EJ, Rowe B, W ard LR. A comparison of multiple drug resistance in salmonellas from humans and food animals in England and W ales, 1981 and 1990. Epidemiol Infect 1993;11:189-197.

44. Glynn KM, Bopp C, D ewitt W, Dabney P, Mokhatar M, Angulo FJ. Emergence of multidrug-resistant Salmonella enterica serotype typhimurium DT104 infections in the United States. N Engl J Med 1998;338: 1333-1338 